\title{
The role of multidetector computed tomography enterography in the evaluation of localized malignant small intestinal lesions: retrospective radiological and pathological experience
}

\author{
Dalia Bayoumi ${ }^{1, A, B, C, D}$, Doaa Khedr ${ }^{1, A, B, F}$, Ahmed Abdallah ${ }^{2, B, C}$, Afaf Taha Ibrahiem ${ }^{3, B, D}$, Sherine Refat ${ }^{3, B, D}$ \\ 'Department of Diagnostic Radiology, Mansoura Faculty of Medicine, Mansoura University, Mansoura, Egypt \\ ${ }^{2}$ Department of surgical oncology, Mansoura Faculty of Medicine, Mansoura University, Mansoura, Egypt \\ ${ }^{3}$ Department of pathology, Mansoura Faculty of Medicine, Mansoura University, Mansoura, Egypt
}

\section{Abstract:}

Purpose: Our purpose is to present our experience in using multidetector computed tomography (MDCT) enterography in the evaluation of localized malignant small intestinal lesions with pathological correlation.

Material and methods: We retrospectively evaluated 53 patients of pathologically proven malignant localized small intestinal tumours, who underwent multidetector CT enterography.

Results: In this study, the mean age was $51.39 \pm 17.4$ years. The most commonly affected age group was from 50 to 59 years. The commonest clinical complaint was abdominal pain. The ileum was the most commonly affected anatomical region, showing 25 lesions (47.16\%). Radiologically irregular/asymmetric wall thickening was detected in 42 cases (79.24\%). Pathologically the most common malignancy was small intestinal adenocarcinoma, followed by carcinoid tumour, lymphoma, and gastrointestinal stromal tumours (GIST). We found that there was a statistically significant association between the pathological lymphadenopathy $(p=0.005)$ and absent proximal intestinal dilatation $(p=0.01)$ with intestinal lymphoma. Also, there was a statistically significant association between the extra-intestinal mesenteric fat changes with carcinoid tumours $(p=0.001)$. Irregular/asymmetric wall thickening was detected in 14 cases of small intestinal adenocarcinoma with a statistically significant association $(p=0.001)$ while exophytic pathological mass formation was statistically significant associated $(p \leq 0.001)$ with small intestinal GIST.

Conclusions: Multidetector CT enterography is a non-invasive and accurate method in the evaluation of focal and localized small intestinal malignant lesions. The accurate detection of these lesions depends to some degree on the experience of the radiologist, lesional size, site and pattern of enhancement, as well as adequate intestinal distension.

Key words: multidetector CT enterography, localized small intestinal lesions, small intestinal malignant tumours.

\section{Introduction}

Malignant small bowel tumours represent only 1-3\% of all gastrointestinal malignancies. Their predisposing factors are different, and they may be hereditary, acquired, or environmental. Unfortunately, because of the absent or non-specific symptoms, they are usually diagnosed late or in their locally advanced stages [1]. The most frequent malignant small intestinal tumours are intestinal adenocarcinoma, neuroendocrinal tumours, lymphomas, and gastrointestinal stromal tumours (GIST) [2].

The small intestine is the longest part of the digestive system, which has a serpentine course, its length is about 20 feet, and it presents about $75 \%$ of the gut length and about $90 \%$ of the gut surface [3]. That is why the imag-

Correspondence address:

Dalia Bayoumi, Department of Diagnostic Radiology, Mansoura Faculty of Medicine, Mansoura University, Mansoura, Egypt, e-mail: daliabayoumi1982@gmail.com

Authors' contribution:

A Study design - B Data collection · C Statistical analysis · D Data interpretation - E Manuscript preparation · F Literature search - G Funds collection 
ing of the small bowel is very challenging technically and requires a large field of view and accurate multiplanar reformatting [4].

The proper diagnosis of small intestinal lesions usually requires multiple modalities including endoscopy and multi-detector CT with adequate bowel distension and good patient preparation. However, in a minority of cases, these diagnostic procedures may yield negative outcomes, so surgical laparoscopy/laparotomy may become inevitable $[5,6]$.

Currently, the availability of multidetector computed tomography (MDCT) has greatly expanded the utility of CT for evaluating small-bowel diseases. MDCT is now readily available, and it can show the whole length and course of the small bowel [7]. MDCT enterography is a dedicated technique that allows proper visualization of the entire small bowel wall to detect abnormal mural thickening and analyse its type, length, and pattern of enhancement [8].

MDCT enterography also helps in the differentiation between inflammatory and non-inflammatory bowel lesions and in the identification of a transition zone in cases of intestinal obstruction. Moreover, MDCT gives an excellent assessment of the extra-enteric structures, nodal status, and distant metastasis $[9,10]$.

Types of abnormal small intestinal thickening include focal or localized thickening, which is less than $5 \mathrm{~cm}$, segmental intestinal thickening, which is from $6 \mathrm{~cm}$ to 40 $\mathrm{cm}$, and diffuse intestinal thickening, which is more than $40 \mathrm{~cm}[11,12]$. MDCT enterography has been efficiently used to evaluate segmental and diffuse intestinal involvement, which are mainly seen in inflammatory bowel disorders, while the detection and characterization of localized small intestinal lesions are more difficult because of the long length and overlapping criteria of the small bowel loops $[13,14]$.

The objective of this study is to present our experience in using MDCT enterography in the evaluation of localized malignant small intestinal lesions with pathological correlation.

\section{Material and methods}

This study was approved by our institutional review board, and informed consent was waived. It is a retrospective study that was conducted at the Radiology Department of Mansoura University and Mansoura University Oncology Centre between September 2017 and December 2019. We searched the institutional electronic database for patients with pathologically confirmed small intestinal malignant masses. We included the patients with localized malignant small bowel lesions who underwent a triphasic scan with adequate CT enterography technique. Patients with signs of peritoneal or metastatic diseases were excluded, as well as patients with improper CT enterography technique.
We included 53 patients ( 32 males and 21 females); their age ranged from 20 to 79 years and the mean \pm SD was $51.39 \pm 17.4$ years. Patients were presented with the following symptoms: abdominal pain, loss of weight, abdominal distension, melena, and intestinal obstruction. We also assessed the following:

- relevant clinical data: including age, sex, and clinical complaints;

- radiological assessment: multidetector CT was performed using commercially available Philips CT with 128 detector rows, Philips with 64 detector rows, and Toshiba CT with 128 detector rows;

- MDCT enterography technical considerations.

MDCT enterography of the small intestine is a complex non-invasive examination that requires good patient preparation and cooperation. The MDCT enterography imaging protocol must include thin slice thickness and adequate detector collimation (to get the advantages of multiplanar reformatting) $[7,8]$.

\section{Patient preparation}

Patients were fasting at least 4 hours before starting the examination. The patients were asked to ingest a low-fibre diet, plenty of fluids, and laxatives at least 24 hours before the examination. In this exam, we used 2 types of contrast material.

\section{Oral water contrast}

MDCT enterography can be done by using negative or neutral oral contrast materials that possess attenuation values close to that of water. There are different types of negative or neutral oral contrast materials, and they include water, water-methylcellulose solution, polyethylene glycol (PEG) electrolyte solution or low-concentration barium, mannitol solution, and low-density barium sulphate preparations (Volumen, $0.1 \% \mathrm{~W} / \mathrm{V}$ ), which allow luminal dilatation and reduce resorption of water through the intestinal wall. The attenuation value of the low-concentration barium is only $20 \mathrm{HU}$. This intraluminal attenuation value must be low enough to allow proper evaluation of normal and abnormal bowel wall enhancement $[9,10]$.

In this study, the patients were asked to ingest 1500 $2000 \mathrm{ml}$ of oral contrast over 30-60 min to achieve adequate luminal distension because collapsed bowel loops can be mistakenly diagnosed as a pathological lesion.

The patients were given 3 bottles of Volumen $(450 \mathrm{ml}$ each) allowing 15 minutes of spacing between them with a glass of water given 5 minutes just before starting the examination to distend the stomach.

\section{IV contrast agents}

IV contrast agents are given according to the patient's body mass index and renal function (after exclusion of other contraindications to contrast material). 
Using an automatic power injector, a dose of 80-120 cc of low osmolar iodinated intravenous contrast agent (U1travist or Omnipaque) was injected at $5 \mathrm{ml} / \mathrm{s}$ with a scan delay of 50-70 s.

The use of intravenous injection of contrast medium is important for the assessment of bowel wall enhancement pattern and evaluation of mesenteric vessels [11].

In the early arterial phase, we can get the best contrast between the enhancing intestinal lesions and the lumen filled with negative contrast material [12].

\section{Image acquisitio}

Images are obtained with a slice thickness of about $1 \mathrm{~mm}$ $\times 0.5 \mathrm{~mm}$ with $1 \mathrm{~mm} \times 1 \mathrm{~mm}$ for reconstruction. We used $120 \mathrm{kV}$ and $250 \mathrm{~mA}$. The field of view (FOV) extended from the level of the diaphragm to the level of the symphysis pubis.

\section{Image analysis and post-processing:}

Thin slices were sent to an independent automatic 3-dimensional workstation where multiplanar reformatted (MPR) and maximum-intensity-projection (MIP) images were generated.

All small bowel abnormalities were evaluated according to the following criteria:

- location of the lesion within the small bowel,

- the pattern of enhancement and its homogeneity,

Table 1. Demographic data and clinical presentation of the study patients

Parameter
\begin{tabular}{|l|c|} 
Sex & $n(\%)$ \\
\hline Male & $32(60.37)$ \\
\hline Female & $21(39.62)$ \\
\hline Age (years) & $5(9.43)$ \\
\hline $20-29$ & $7(13.2)$ \\
\hline $30-39$ & $13(24.52)$ \\
\hline $40-49$ & $16(30.18)$ \\
\hline $50-59$ & $8(15.09)$ \\
\hline $60-69$ & $4(7.54)$ \\
\hline $70-79$ & $51.39 \pm 17.4$ \\
\hline Mean \pm SD & \\
\hline Complaints & $53(100.00)$ \\
\hline Abdominal pain & $26(49.05)$ \\
\hline Anorexia & $13(24.52)$ \\
\hline Vomiting & $12(22.64)$ \\
\hline Body mass loss & $10(18.86)$ \\
\hline Constipation & $8(15.09)$ \\
\hline Bleeding & $6(11.32)$ \\
\hline Jaundice & \\
\hline
\end{tabular}

- length of involvement,

- the type of bowel wall thickening and whether it is regular or irregular,

- extraintestinal findings,

- associated abnormalities in the mesentery,

- special findings of different disease entities.

\section{Data verification}

Operative findings and/or biopsy results were obtained.

\section{Statistical analysis}

We analysed the data using SPSS version 21 . The normality of data was first tested with a one-sample KolmogorovSmirnov test. Qualitative data were described using the number and percentage while continuous variables were presented as mean \pm SD (standard deviation).

\section{Results}

In this study 53 patients were included. We had 32 males $(60 \%)$ and 21 females (40\%). Their ages ranged from 20 years to 79 years and the mean age (mean \pm SD) was 51.39 \pm 17.4 years. The most commonly affected age group was from 50 to 59 years, representing about 16 cases (30.18\%), followed by the age group from 40 to 49 years, representing about 13 cases $(24.52 \%)$. The most common clinical complaints were abdominal pain in all cases 53 cases $(100 \%)$ and anorexia in 26 cases (49.05\%) (Table 1$)$.

In the baseline characteristics of the lesions, the ileum was the most commonly affected anatomical region, showing 25 lesions (47.16\%) followed by the duodenum - 16 lesions (30.18\%) and the jejunum - 12 lesions (22.64\%). Radiologically irregular/asymmetric wall thickening was detected in 42 cases (79.24\%), and regular/symmetric wall thickening was detected in 11 cases (20.75\%). While exophytic mass formation was detected in 16 cases $(30.18 \%)$ and was absent in the remaining 37 cases (69.81\%) (Table 2).

Table 2. Baseline characteristics of the lesions

\begin{tabular}{|c|c|}
\hline Parameter & $n(\%)$ \\
\hline \multicolumn{2}{|l|}{ Site } \\
\hline Jejunum & $12(22.64)$ \\
\hline Duodenum & $16(30.18)$ \\
\hline lleum & $25(47.16)$ \\
\hline \multicolumn{2}{|l|}{ Type of involvement } \\
\hline Irregular/Asymmetric & $42(79.24)$ \\
\hline Regular/Symmetric & $11(20.75)$ \\
\hline \multicolumn{2}{|l|}{ Exophytic mass formation } \\
\hline Yes & $16(30.18)$ \\
\hline No & $37(69.81)$ \\
\hline
\end{tabular}


Table 3. Extra-lesional findings

Extraintestinal findings
\begin{tabular}{|l|l|}
\hline \multicolumn{2}{|l|}{ Suspicious lymphadenopathy } \\
\hline Yes & $36(67.92)$ \\
\hline No & $17(32.07)$ \\
\hline \begin{tabular}{l|l|} 
Mesenteric changes \\
\hline Yes
\end{tabular} \\
\hline No & $30(56.6)$ \\
\hline Proximal obstruction & $23(41.5)$ \\
\hline Yes & \\
\hline No & $28(52.83)$ \\
\hline
\end{tabular}

The secondary extra-lesional findings, are suspicious lymphadenopathy, which was found in 36 cases $(66.03 \%)$ and absent in 17 cases $(32.07 \%)$; mesenteric changes were detected in 30 cases (56.6\%) and absent in 22 cases (41.5\%); and proximal obstruction was found in 28 cases (52.83\%) and absent in 25 cases (47.16\%) (Table 3 ).

Regarding the pathological types identified in our study, there were 15 cases $(28.3 \%)$ of small intestinal adenocarcinoma, all of which were located in the duodenum; 10 of them were of the undifferentiated type, 3 of moderately differentiated type, and 2 of well-differentiated type. We had
Table 4. Pathological types of the lesions

\begin{tabular}{|l|c|}
\hline Pathological type & $n(\%)$ \\
\hline Adenocarcinoma & $15(28.30)$ \\
\hline Carcinoid & $14(26.41)$ \\
\hline Lymphoma & $13(24.52)$ \\
\hline GIST & $11(20.75)$ \\
\hline
\end{tabular}

Table 5. Pathological types, sites, and percentages of each lesion

\begin{tabular}{|l|l|c|}
\hline Pathological type & Site of involvement & $\%$ \\
\hline Adenocarcinoma & Duodenum (15 cases) & -28.30 \\
\hline \multirow{2}{*}{ Carcinoid tumour } & lleum (12 cases) & -22.64 \\
\cline { 2 - 3 } & $\begin{array}{l}\text { Jejunum and duodenum (2 cases, } \\
1 \text { case each) }\end{array}$ & -3.77 \\
\hline \multirow{2}{*}{ Lymphoma } & lleum (10 cases) & -18.86 \\
\cline { 2 - 3 } & Jejunum (3 cases) & -5.66 \\
\hline \multirow{2}{*}{ GIST } & lleum (3 cases) & -5.66 \\
\cline { 2 - 3 } & Jejunum (8 cases) & -15.09 \\
\hline
\end{tabular}

14 cases $(26.41 \%)$ of neuroendocrine (carcinoid) tumour; 12 lesions were located in the ileum and 2 lesions were located in the jejunum and the duodenum. We also had

Table 6. The association between pathological types of the lesions and radiological findings (total number of cases 53)

\begin{tabular}{|c|c|c|c|c|}
\hline Radiological findings & $\begin{array}{l}\text { Adenocarcinoma } \\
\qquad n=15\end{array}$ & $\begin{array}{c}\text { Carcinoid } \\
n=14\end{array}$ & $\begin{array}{l}\text { Lymphoma } \\
n=13\end{array}$ & $\begin{array}{c}\text { GIST } \\
n=11\end{array}$ \\
\hline \multicolumn{5}{|l|}{ Lymphadenopathy } \\
\hline Yes $(n=36)$ & $12(80.0)$ & $6(42.9)$ & $13(100.0)$ & $5(45.5)$ \\
\hline № $(n=17)$ & $3(20.0)$ & $8(57.1)$ & $0(0.0)$ & $6(54.5)$ \\
\hline$p$-value & 0.237 & $0.019^{*}$ & $0.005^{*}$ & 0.07 \\
\hline \multicolumn{5}{|l|}{ Mesenteric changes } \\
\hline Yes $(n=30)$ & $8(53.3)$ & $14(100.0)$ & $1(7.7)$ & $7(63.6)$ \\
\hline No $(n=23)$ & $7(46.7)$ & $0(0.0)$ & $12(92.3)$ & $4(36.4)$ \\
\hline$p$-value & 0.763 & $0.001^{*}$ & $0.0004^{*}$ & 0.59 \\
\hline \multicolumn{5}{|l|}{ Wall thickening } \\
\hline Regular $(n=11)$ & $1(6.7)$ & $4(18.6)$ & $5(38.5)$ & $1(9.1)$ \\
\hline Irregular $(n=42)$ & $14(93.3)$ & $10(71.4)$ & $8(61.5)$ & $10(90.9)$ \\
\hline$p$-value & $0.001^{*}$ & 0.089 & 0.07 & 0.283 \\
\hline \multicolumn{5}{|l|}{ Exophytic mass formation } \\
\hline Yes $(n=16)$ & $2(13.3)$ & $1(7.1)$ & $2(15.4)$ & $11(100.0)$ \\
\hline No $(n=37)$ & $13(86.7)$ & $13(92.9)$ & $11(84.6)$ & $0(0.0)$ \\
\hline$p$-value & 0.093 & $0.028^{*}$ & 0.093 & $<0.001^{*}$ \\
\hline \multicolumn{5}{|l|}{ Proximal dilatation } \\
\hline Yes $(n=28)$ & $9(60.0)$ & $8(57.1)$ & $3(23.1)$ & $8(72.7)$ \\
\hline No $(n=25)$ & $6(40.0)$ & $6(42.9)$ & $10(76.9)$ & $3(27.3)$ \\
\hline$p$-value & 0.511 & 0.706 & $0.01^{*}$ & 0.137 \\
\hline
\end{tabular}


13 cases $(24.52 \%)$ of B-cell lymphoma; 10 were located in the ileum and 3 were located in the jejunum. Also, we had 11 cases $(20.75 \%)$ of GIST; 6 were of malignant type and 5 of benign type; 3 were located in the ileum and 8 were located in the jejunum (Tables 4 and 5).

The association between the different pathological types and radiological findings are shown in Table 6. We found that there was a statistically significant association between the pathological lymphadenopathy $(p=0.005)$ and absent proximal intestinal luminal dilatation $(p=0.01)$ with intestinal lymphoma. Also, there was a statistically significant association between the extra-intestinal mesenteric fat changes and carcinoid tumours $(p=0.001)$. Irregular/asymmetric wall thickening was detected in 14 cases of small intestinal adenocarcinoma with a statistically significant association ( $p=0.001)$, while exophytic pathological mass formation was statistically significantly associated $(p \leq 0.001)$ with small intestinal GIST.

\section{Discussion}

In this study, the most commonly affected age group was between 50 and 59 years (16 cases, $30.18 \%$ ); this was in agreement with many studies such as Shinya et al. [15] and Kim et al. [16], who stated that most of the neoplastic small intestinal lesions were common in older age groups, especially in the fifth and sixth decades of life, and that the age peak of each tumour differs according to the specific pathological type.

Regarding the clinical presentations of the patients, different clinical complaints were encountered in this study; the most common complaint was abdominal pain, which was seen in all 53 cases included in this study, followed by anorexia, vomiting, weight loss, constipation, and bleeding per rectum in order of frequency. Laghi et al. [17] and Rengo et al. [18], stated that patients with small bowel diseases in general and small bowel malignant lesions in particular usually present with vague symptoms such as abdominal pain, signs of acute or sub-acute intestinal obstruction, bleeding, anorexia, weight loss, signs of bowel perforation, or even jaundice.

The clinical presentation of small intestinal diseases varies widely according to the site and the pathological type of the lesions. In intestinal lymphomas, patients may present with non-specific symptoms such as abdominal pain, vomiting, nausea, and large-sized abdominal masses with the characteristic central aneurysmal intestinal dilatation, which delays the onset of intestinal obstruction. [16]. In contrast to lymphoma, small lesions of intestinal adenocarcinoma can result in early signs of intestinal obstruction or even perforation of the intestinal lumen, while in GIST intestinal obstruction is usually delayed because most of these tumours grow in an exophytic direction allowing the pathological masses to attain large sizes before the development of intestinal obstruction. The GIST patients usually suffer from bleeding due to recurrent mucosal ulcerations, but they can present also with a wide range of other symptoms like abdominal pain, nausea, and vomiting. Some types of diarrhoea and hormonal disturbances are common with carcinoid tumours [18].

Karaosmanoglu et al. [3] mentioned that pathological small bowel wall thickness usually exceeds $3 \mathrm{~mm}$, and diffuse regular circumferential intestinal mural thickening is almost detected in non-neoplastic and inflammatory small bowel diseases, with a few exceptions, while irregular asymmetric intestinal mural thickening characterizes neoplastic lesions. In our study 42 (79.24\%) out of 53 cases of malignant lesions had irregular localized asymmetric bowel wall thickening. The remaining 11 cases $(20.75 \%)$ that showed regular symmetric small bowel wall thickening comprised 1 case of well-differentiated adenocarcinoma, 5 cases of low-grade lymphoma, 4 cases of carcinoid tumours, and 1 case of GIST. These results are in agreement with Antonakou et al. [19], who stated that possible regular symmetric mural or mucosal thickening could be depicted in some malignant tumours in their early stages and some low-grade malignancies as well-differentiated adenocarcinoma and low-grade lymphoma.

Regarding the sites of affection, in this study, the ileum was the most commonly affected region (25 cases, $47.16 \%$ ) followed by the duodenum (16 cases, $30.18 \%$ ) and jejunum (12 cases, 22.64\%). These sites of affection differ according to the pathological type of lesion [4].

In this study, we had 15 cases $(28.3 \%)$ of intestinal adenocarcinoma (Figure 1). All of these lesions were located in the duodenum, and they were presented as an irregular asymmetric intestinal thickening except 1 case of well-differentiated adenocarcinoma, which was presented as regular symmetric thickening. Other positive secondary findings included metastatic lymphadenopathy in 12 cases, mesenteric changes in 8 cases, exophytic lesions in 2 cases, and proximal intestinal dilatation in 9 cases. These results are in agreement with Nowel et al. [20], who reported that primary small intestinal adenocarcinoma is a relatively rare tumour (representing less than $2 \%$ of all GI neoplasms), but it is the commonest primary small bowel malignancy. The most frequent site for these tumours is the duodenum, followed by the jejunum and the ileum.

Small intestinal adenocarcinoma can develop secondary to other pathologies such as Crohn's disease and Celiac disease. Typical imaging appearance includes irregular asymmetric intestinal thickening with heterogeneous contrast enhancement $[21,22]$.

Also, our study was in agreement with Weber et al. [23], who stated that circumferential irregular and annular luminal narrowing is a common presentation, usually resulting in serious complications such as intussusception or bowel obstruction, while ampullary tumours are malignant intestinal tumours arising in the duodenum, resulting in common bile duct (CBD) obstruction and secondary obstructive jaundice [24]. 

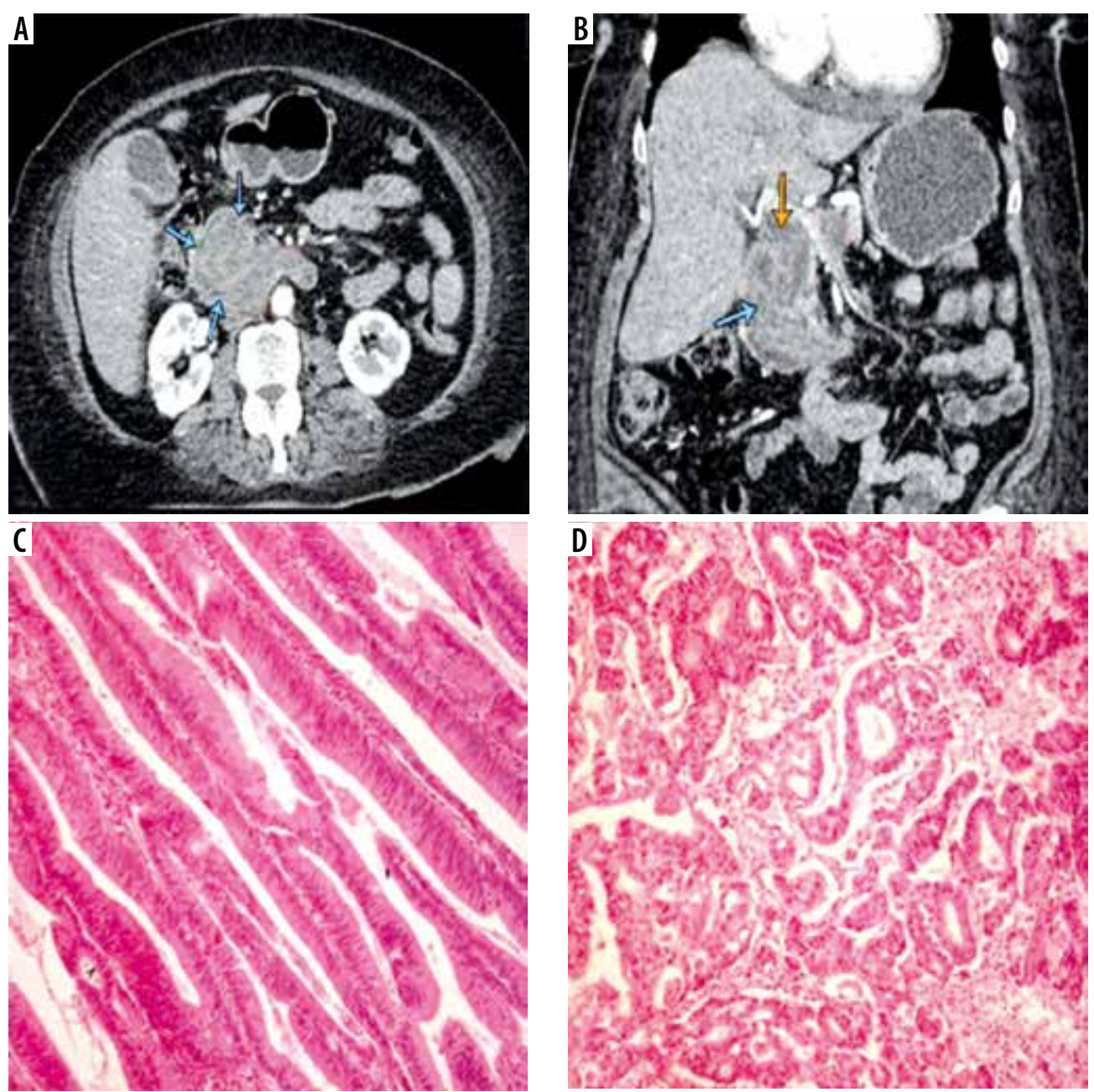

Figure 1. Duodenal adenocarcinoma. Axial (A) and reformatted coronal (B) multidetector computed tomography enterography images showing an ill-defined irregular mural thickening forming heterogeneous soft tissue mass (marked by the blue arrows) in the duodenum at the ampullary region compressing the distal common bile duct (marked by the red arrow). Pathologically there is villous adenoma of small intestine with dysplastic features $(\mathbf{C}, \times 400)$ however in photo $D$, there is Gll invasive adenocarcinoma with more complex and cribriform arrangement of neoplastic glands $(D, \times 400)$

In this study, we had (14 cases, $26.41 \%)$ of small intestinal carcinoid; 12 cases $(22.64 \%)$ were located in the ileum and 2 cases $(3.77 \%)$ in the jejunum and in the duodenum. Regarding the radiological appearance of carcinoid tumours, we encountered 2 types: 8 cases (15.09\%) were presented as hyper-enhancing mural or intraluminal intestinal lesion of variable size (Figure 2), and the other radiological appearance detected was irregular mesenteric masses in 6 cases $(11.32 \%)$ surrounded by desmoplastic reaction/ fibrotic bands attracting the surrounding intestinal loops (Figure 3), which showed irregular asymmetric intestinal thickening (in 10 cases) and regular symmetric intestinal thickening (in 4 cases). Other positive secondary findings in this category were metastatic lymphadenopathy in 6 cases, exophytic lesion in 1 case, and proximal intestinal dilatation in 8 cases.

Carcinoid tumours are the second most common category of primary small intestinal malignant tumours; about $30 \%$ of GIT neuroendocrine tumours arise from the small intestine [5]. Our results are in agreement with Baxi et al. [25] regarding the site and radiological appearance of car- cinoid tumours; they reported that the ileum is the most common site for carcinoid tumours and that the MDCT enterography can show polypoidal avidly enhancing intestinal lesions of variable size that may be single or multiple, associated with distortion of the small bowel course, and give the characteristic "hairpin" kinks of the small bowel. Intra-tumoral calcifications may be present in $70 \%$ of cases.

Other radiological appearances of small bowel carcinoid tumours include enhancing mesenteric mass with radiating bands eliciting fibrosis and desmoplastic response attracting the adjacent small bowel loops suggesting infiltration. The liver is the most common site for metastatic lesions, which are usually hypervascular lesions. In this study all the lesions were identified early before the development of hepatic metastatic deposits [26].

In this study we had 13 cases $(24.52 \%)$ of small intestinal lymphoma (Figure 4$) ; 10$ cases $(18.86 \%)$ were located in the ileum, and 3 cases $(5.66 \%)$ in the jejunum - all of these cases were pathologically proven non-Hodgkin Bcell lymphoma. Radiologically there was irregular asymmetric intestinal thickening detected in 8 cases and regular 

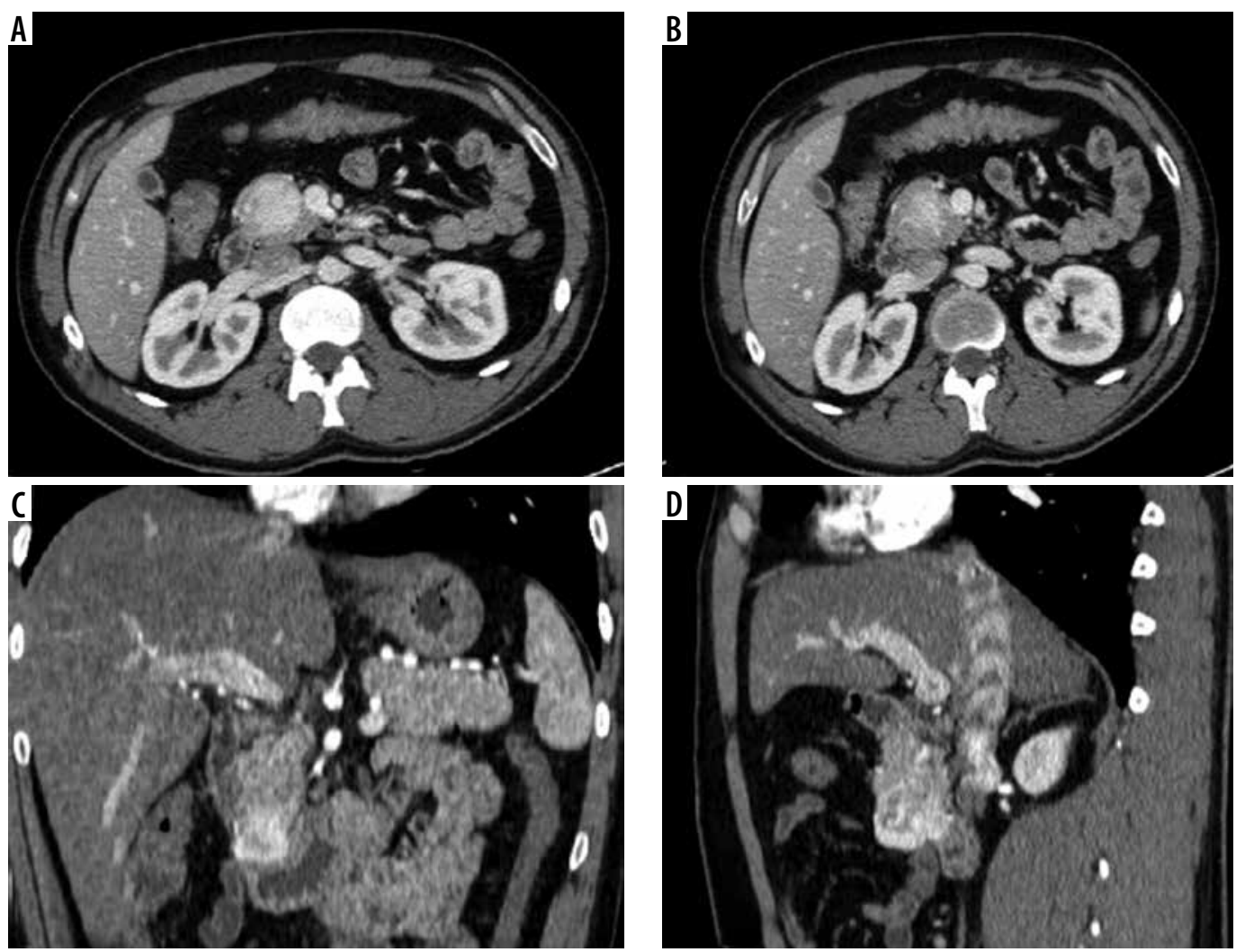

Figure 2. Primary small intestinal neuroendocrinal tumor. Axial (A, B), reformatted coronal (C) and sagittal (D) multidetector computed tomography enterography images showing a well-defined hyper-enhancing mass lesion in the second part of the duodenum, just before inferior flexure. Pathologically proven neuroendocrine tumor
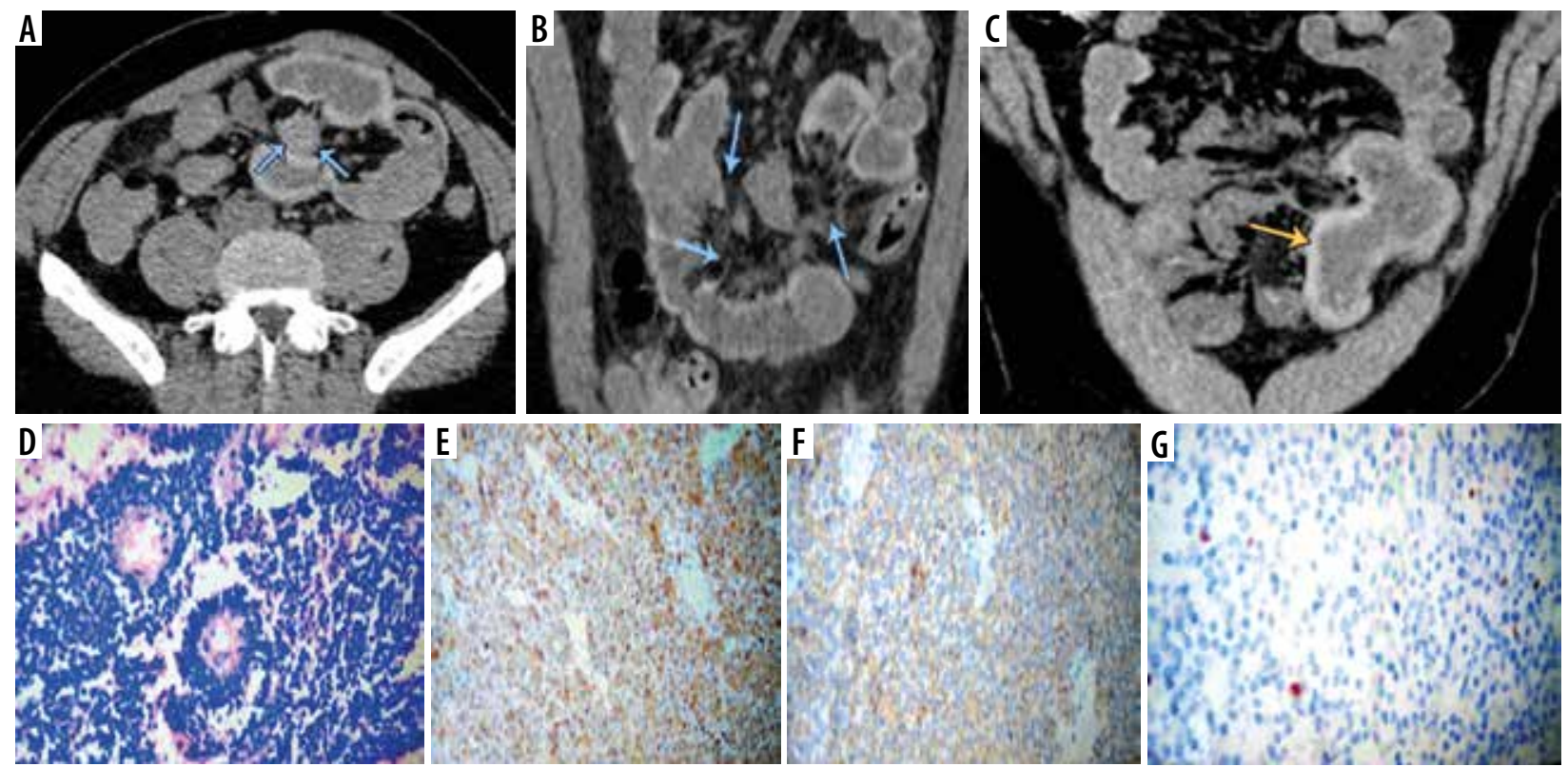

Figure 3. Primary small intestinal carcinoid tumor. Axial (A), reformatted coronal (B) and sagittal (C) multidetector computed tomography enterography images showing a well-defined speculated mesenteric mass with surrounding fibrotic reaction, dirtiness of fat planes (marked by the blue arrows) and traction of the small bowel (ileal) loops that showed irregular circumferential mural thickening (marked by the yellow arrow). Pathologically, there are monotonous small cells arranged in trabecular and perivascular pattern $(D, \times 200 \mathrm{H} \& \mathrm{E})$. Diffuse positive cytoplasmic staining for chromogranin and synptophysin $(E, \times 400 ; F, \times 400)$ respectively and low expression for Ki $67(G, K i 67)$

symmetric intestinal thickening in 5 cases. Other positive secondary findings included pathological lymphadenopathy in all 13 cases, exophytic lesions in 2 cases, and proximal intestinal dilatation in 3 cases.
Lewis et al. [27], Manning et al. [28], and Yang et al. [29] reported that lymphoma is the third most common small intestinal neoplastic lesion. Pathologically most of the cases are non-Hodgkin B-cell lymphoma. Other types of 

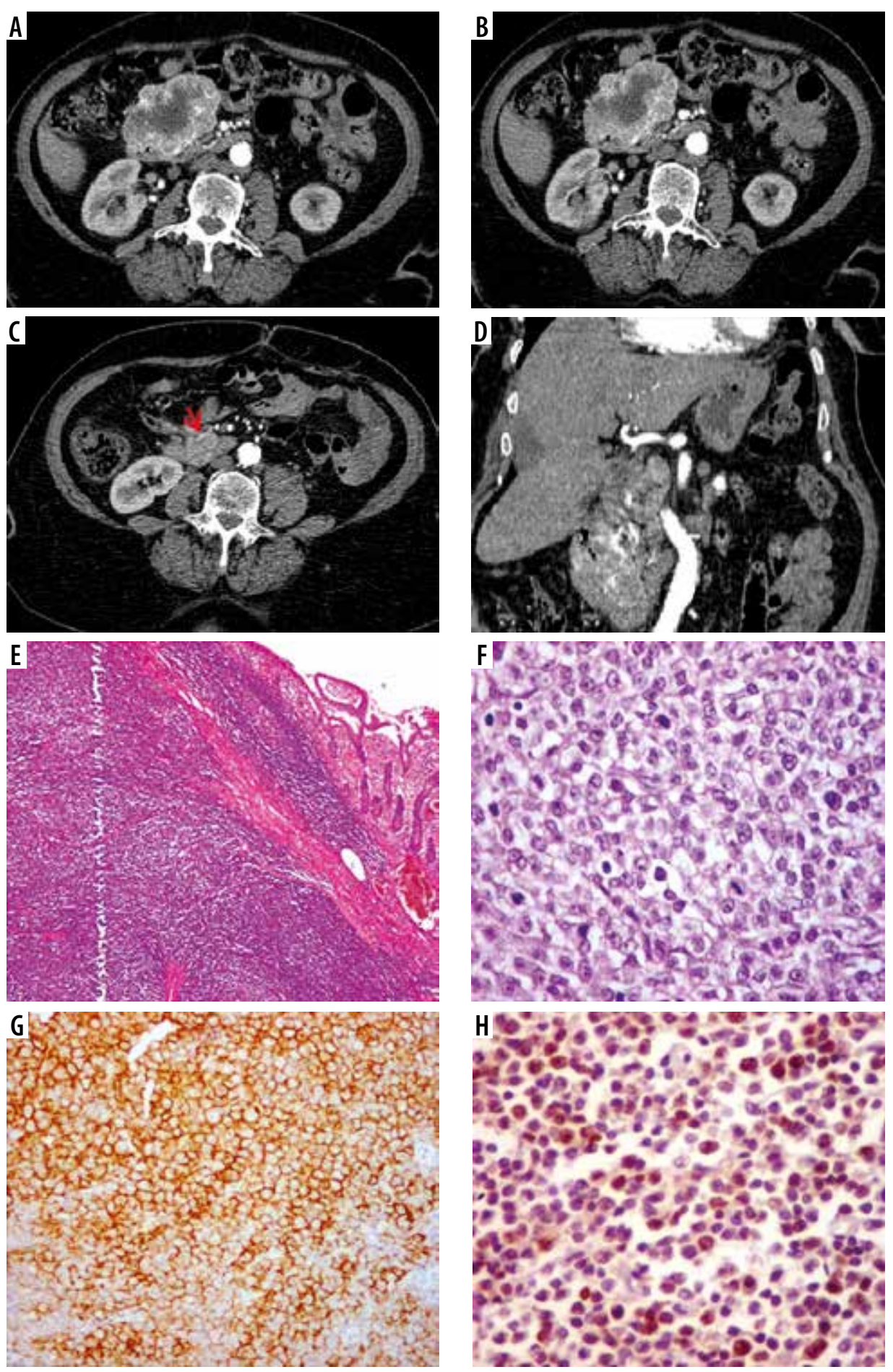

Figure 4. Primary small intestinal B-cell lymphoma. Axial (A, B, C) and reformatted coronal (D) multidetector computed tomography enterography images showing an ill-defined irregular mural thickening forming heterogeneous intestinal soft tissue mass with malignant regional lymphadenopathy (marked by the red arrow). Pathologically there is dense lymphocytic infiltration of the submucosa and musculosa of small intestine (E x100, H\&E), higher magnification revealed large cells with vesicular nuclei and prominent nucleoli and frequent mitoses ( $\mathrm{F} \times 400, \mathrm{H \& E}$ ). The cells showed diffuse expression of CD20 and high Ki67 proliferation index $(\mathrm{G} \times 400)$ and $(\mathrm{H} \times 400)$ respectively

lymphoma such as T-cell lymphoma are usually associated with an immunological disease or inflammatory bowel disorders. Burkitt's lymphoma is a special type of lymphoma associated with Epstein-Barr virus infection occurring in infancy at the ileocecal region.

MDCT enterography features of small bowel lymphoma are irregular intestinal wall thickening with mild enhancement most commonly involving the ileum (because it contains the highest amount of lymphoid tissue) with associated pathologically appearing mesenteric lymph nodes. Lymphadenopathy associated with small bowel lymphoma is usually bulky and larger than lymphadenopathy in other small intestinal neoplastic lesions. Proximal bowel obstruction is an uncommon finding in lymphoma due to 

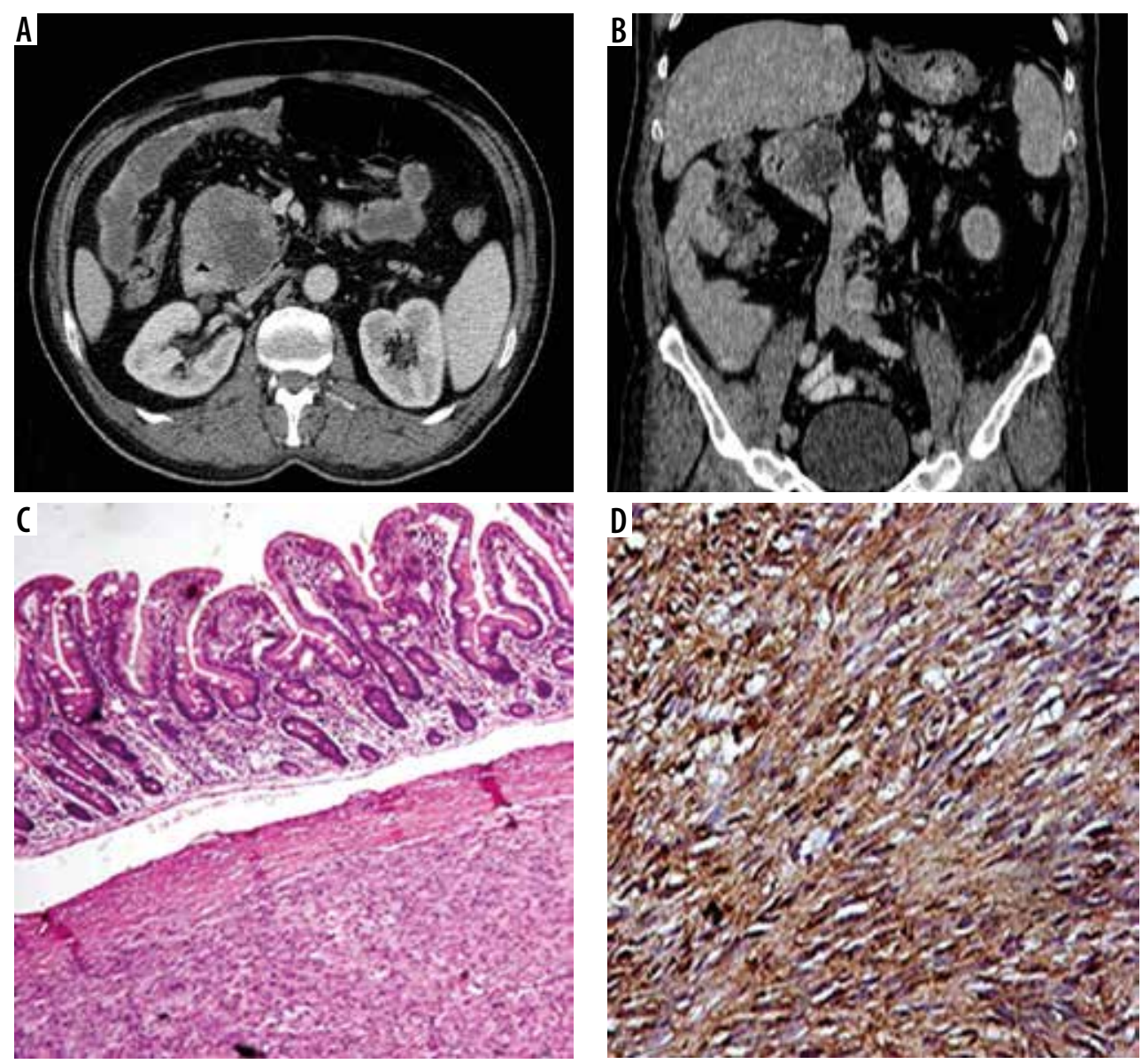

Figure 5. Duodenal gastrointestinal stromal tumour. Axial (A) and reformatted coronal (B) multidetector computed tomography enterography showing predominantly hypodense duodenal mass with exophytic component. Pathologically there is submucosal spindle cell proliferation surrounded by pseudocapsule $(C, \times 100)$, with positive diffuse staining for CD 117 (D, CD117)

the pathognomonic aneurysmal intestinal dilatation of the affected lumen in cases of lymphoma (in this study, mild proximal intestinal dilatation was detected only in 3 cases of lymphoma) [30].

Other forms of the disease include nodular mucosal thickening and exophytic masses. Lymphoma can also involve a long segment of small intestine with possible ulceration, necrosis, and perforation. Focal lymphomatous masses involving the terminal ileum can mimic Crohn's disease or even act as a head for intussusception. Splenomegaly and non-regional lymphadenopathy should not be overlooked because they represent the cardinal signs supporting the diagnosis of lymphoma [29].

In this study, we had 11 cases $(20.75 \%)$ of small bowel gastrointestinal stromal tumours (Figure 5); 3 cases (5.66\%) were located in the ileum, and 8 cases $(15.09 \%)$ in the jejunum. Radiologically irregular asymmetric intestinal thickening was detected in 10 cases, and exophytic mass formation was detected in all cases. Other positive secondary findings included pathological lymphadenopathy in all 5 cases and proximal intestinal dilatation in 8 cases.

Baheti et al. [31] and Koc et al. [32] reported that GIST are the most common mesenchymal intestinal tumours affecting patients above 40 years of age, and they include both benign and malignant varieties. These tumours most fre- quently involve the submucosal region, and then they can project into the intestinal lumen or grow in an exophytic direction.

MDCT and MDCT enterography in cases of GIST usually show large-sized hyper-enhancing masses with heterogeneous appearance resulting from tissue necrosis or intra-tumoral haemorrhage in addition to the asymmetric localized or segmental intestinal wall thickening. The role of MDCT enterography is also highly valuable in identifying small-sized lesions that are almost obscured by the collapsed or inadequately opacified intestinal loops [33].

There are some limitations to this study. First, the number of patients is relatively small because they were confined to patients who had pathologically proven malignant small intestinal lesions with adequate MDCT enterography technique only. So, we recommend further studies with a larger sample size. Second, we admit that it would have been better to examine the patients also by PET/CT or by capsule endoscopy to compare the results of these techniques with MDCT to improve the outcome, especially with small-sized intestinal masses. Finally, short focal areas of small intestinal peristaltic motions may sometimes be incorrectly diagnosed as iso- or hyper-enhancing lesions. So, radiologists must confirm the presence of pathological lesions in all planes and phases of the MDCT enterography study. 


\section{Conclusions}

Multidetector CT enterography is a non-invasive and accurate method in the evaluation of focal and localized small intestinal malignant lesions. The detection of these lesions depends to some degree on the experience of the radiologist, lesional size, site and pattern of enhancement, as well as adequate intestinal distension.

\section{Conflicts of interest}

The authors report no conflict of interest.

\section{References}

1. Williams EA, Bowman AW. Multimodality imaging of small bowel neoplasms. Abdom Radiol (NY) 2019; 44: 2089-2103.

2. Agarwala R, Singh AK, Shah J, et al. Ileocecal thickening: clinical approach to a common problem. JGH Open 2019; 3: 456-463.

3. Karaosmanoglu AD, Onur MR, Arellano RS. Imaging in gastrointestinal cancers. In: Textbook of Gastrointestinal Oncology. Springer, Cham 2019; 445-464

4. Jasti R, Carucci LR. Small bowel neoplasms: a pictorial review. Radiographics 2020. DOI: https://doi.org/10.1148/rg.2020200011.

5. Sokhandon F, Al-Katib S, Bahoura L, et al. Multidetector CT enterography of focal small bowel lesions: a radiological-pathological correlation. Abdom Radiol (NY) 2017; 42: 1319-1341.

6. Dillman JR, Adler J, Zimmermann EM, Strouse PJ. CT enterography of pediatric Crohn disease. Pediatr Radiol 2010; 40: 97.

7. Ilangovan R, Burling D, George A, et al. CT enterography: review of technique and practical tips. Br J Radiol 2012; 85: 876-886.

8. Elsayes KM, Al-Hawary MM, Jagdish J, et al. CT enterography: principles, trends, and interpretation of findings. Radiographics 2010 30: 1955-1970.

9. Paulsen SR, Huprich JE, Fletcher JG, et al. CT enterography as a diagnostic tool in evaluating small bowel disorders: review of clinical experience with over 700 cases. Radiographics 2006; 26: 641-657.

10. Macari M, Megibow AJ, Balthazar EJ. A pattern approach to the abnormal small bowel: observations at MDCT and CT enterography. Am J Roentgenol 2007; 188: 1344-1355.

11. Patel KK, Khandhedia MV, Bhardava VH. Characterization of bowel lesions with multi-detector CT scan. Int J Contemp Med Surg Radiol 2018; 3: C136-C142.

12. Tapasvi C, Prajapati N, Madhok R, et al. Evaluation of bowel wall thickening by computed tomography to differentiate benign from malignant lesions. J Clin Diagn Res 2014; 8: RC09-RC12.

13. Fernandes T, Oliveira MI, Castro R, et al. Bowel wall thickening at CT: simplifying the diagnosis. Insights Imaging 2014; 5: 195-208.

14. Ghimire Rayamaj K, Peng P, Guo M, Zhong D. Computed tomography: a reliable modality for the assessment of bowel wall thickening to categorize benign and malignant lesions of bowel wall. J Mol Imag Dynamic 2018; 8: 2.

15. Shinya T, Inai R, Tanaka T, et al. Small bowel neoplasms: enhancement patterns and differentiation using post-contrast multiphasic multidetector CT. Abdom Radiol (NY) 2017; 42: 794-801.

16. Kim JS, Park SH, Hansel S, Fletcher JG. Imaging and screening of cancer of the small bowel. Radiol Clin 2017; 55: 1273-1291.

17. Laghi A, Hara AK. Small bowel disease. In: Diseases of the Abdomen and Pelvis. Springer, Cham 2018; 117-122.

18. Rengo M, Picchia S, Laghi A. Small bowel MDCT. In: Multislice CT. Springer, Cham 2017; 619-639.
19. Antonakou I, lazaridou E, Stefanou D, et al. Bowel wall thickness, which underlying pathology can be masqueraded? European Congress of Radiology 2020; poster no: C-14123. DOI: https://dx.doi. org/10.26044/ecr2020/C-14123.

20. Nowell KA, Howe JR. Small bowel adenocarcinoma. In: Gastrointestinal Oncology. CRC Press 2016; 121-126.

21. Salem AA, El Shoieby MH, Mourad AF, et al. Surgical and radiological assessment of small intestinal neoplasms. J Cancer Ther 2016; 7: 96.

22. Aydin D, Sendur MA, Kefeli U, et al. Evaluation of prognostic factors and adjuvant chemotherapy in patients with small bowel adenocarcinoma who underwent curative resection. Clin Colorectal Cancer 2017; 16: 220-227.

23. Weber NK, Fletcher JG, Fidler JL, et al. Clinical characteristics and imaging features of small bowel adenocarcinomas in Crohn's disease. Abdom Imaging (NY) 2015; 40: 1060-1067.

24. Suh CH, Tirumani SH, Shinagare AB, et al. Diagnosis and management of duodenal adenocarcinomas: a comprehensive review for the radiologist. Abdom Imaging (NY) 2015; 40: 1110-1120.

25. Baxi AJ, Chintapalli K, Katkar A, et al. Multimodality imaging findings in carcinoid tumors: a head-to-toe spectrum. Radiographics 2017; 37: 516-536.

26. Laval VR, Pavel M, Steffen IG, et al. Mesenteric fibrosis in midgut neuroendocrine tumors: functionality and radiological features. Neuroendocrinology 2018; 106: 139-147.

27. Lewis RB, Mehrotra AK, Rodríguez P, et al. From the radiologic pathology archives: gastrointestinal lymphoma: radiologic and pathologic findings. Radiographics 2014; 34: 1934-1953.

28. Manning MA, Somwaru AS, Mehrotra AK, Levine MS. Gastrointestinal lymphoma: radiologic-pathologic correlation. Radiol Clin 2016; 54: 765-784.

29. Yang CB, Yu N, Jian YJ, et al. Spectral CT imaging in the differential diagnosis of small bowel adenocarcinoma from primary small intestinal lymphoma. Academic Radiology 2019; 26: 878-884.

30. Lo Re G, Federica V, Midiri F, et al. Radiological features of gastrointestinal lymphoma. Gastroenterology Research and Practice 2016; 2016: 2498143. DOI: https://doi.org/10.1155/2016/2498143.

31. Baheti AD, Shinagare AB, O'Neill AC, et al. MDCT and clinicopathological features of small bowel gastrointestinal stromal tumours in 102 patients: a single institute experience. Br J Radiol 2015; 88: 20150085.

32. Koc M, Serhatlioglu S. The investigation of incidence and radiological findings of gastrointestinal stromal tumor as a rare cause of abdominal mass. J Turgut Ozal Med Cent 2017; 24: 301-304.

33. Vasconcelos RN, Dolan SG, Barlow JM, et al. Impact of CT enterography on the diagnosis of small bowel gastrointestinal stromal tumors. Abdom Radiol (NY) 2017; 42: 1365-1373. 